\title{
Aplicaciones de Windows Forms con C\#. Automatización del proceso de facturación para la Librería ABC
}

\author{
C\# Windows forms with applications. The automation \\ of the billing process for Librería $A B C$
}

\author{
Verónica Idalia Rosa-Urrutia' \\ Colaboradores: \\ Jessica Lissette Interiano-Segura² \\ Luis David León-Alejo² \\ Claudia Beatriz Pérez-Hernández ${ }^{2}$ \\ Universidad Tecnológica de El Salvador \\ veronica.rosa@utec.edu.sv

\section{Resumen}

El proyecto de investigación se ha basado en una metodología que expone la actual situación del proceso de facturación que realiza la entidad comercial, mediante el seguimiento de la técnica de la entrevista dirigida al dueño y a sus vendedores. Además, se hizo uso de la técnica de observación para obtener un análisis detallado y claro de los inconvenientes y necesidades que se deben corregir.

La librería $A B C$ necesita de una solución, en paralelo a la era digital en la que vivimos. La utilización de un software que administre y registre cada una de las transacciones generadas por sus ventas es de suma importancia no solo porque ayudaría a la microempresa con sus actividades,

\section{Abstract}

The research project is based on a type of methodology that shows the current situation of the billing process used in the bookstore [Librería $A B C$ ]. Interviews were conducted with the owner and the salespeople. Besides, the observation technique was applied in order to obtain a clear and detailed analysis on the needs and problems that need to be corrected.

Librería $A B C$ needs a solution that is parallel to the digital era we live in. It is very important that they use a software to administer and record each of the transactions generated by its sales, not only because it would help this small business in its activities, but also because it would benefit the research team with

\footnotetext{
Ingeniera en Sistemas y Computación, máster en Docencia Universitaria, máster en Visual Analytics y Big Data, Docente tiempo completo de la Universidad Tecnológica de El Salvador. Agradezco la colaboración de mis estudiantes de Programación I por el trabajo realizado con el proyecto.

2 Ayudantes de investigación
} 
sino también porque beneficia al equipo de investigación con el estudio de temas tales como ingeniería de software, programación en C\# en Visual Studio, programación orientada a objetos y sistemas automatizados para ser aplicados en el proyecto. Por lo tanto, el objetivo de la investigación fue crear un sistema que automatice el proceso de facturación de la librería.

Los resultados obtenidos fueron satisfactorios, porque se logró implementar el proyecto y se le dará seguimiento para nuevos requerimientos. Debido a lo anterior, el aprendizaje obtenido fue la mejor ganancia con la investigación realizada.

\section{Palabras clave}

C\# (Lenguaje de programación de computadores). Programación orientada a objetos (computación). Automatización de oficinas. Procesamiento electrónico de datos.

\section{Introducción}

La librería $A B C$ se fundó el 8 de septiembre del 2015, casualmente el mismo día y mes en que, en 1965, se proclamó por la Organización de las Naciones Unidas para la Educación, la Ciencia y la Cultura como el "Día Internacional de la Alfabetización", con lo cual se busca concientizar al mundo entero sobre la importancia de saber leer y escribir.

Don Carlos Alfaro, junto con su esposa, doña Laura Blanco de Alfaro, deciden iniciar su propio negocio. En el proceso encuentran un socio, don Feliciano Cisneros, quien accede a formar parte en el emprendimiento de sus amigos; y así es como nace la librería $\mathrm{ABC}$, un negocio familiar en donde lo principal es ofrecer una atención y un servicio excelente hacia los clientes.

La librería $A B C$ es una microempresa innovadora y dirigida para satisfacer las necesidades educativas del público en general. Comprometida con hacer sentir a sus clientes, que adquieren un producto de calidad a un precio justo y accesible. La atención en la sucursal se ve reflejada en la calidez humana, en el trato cordial, amable y personalizado hacia las personas. a study on topics such as software engineering, C\# programming in Visual Studio, programming oriented to objects and automated systems to be applied in this project. Therefore, the objective was to create a system that can perform the billing process of the bookstore in an automated manner.

The obtained results were satisfactory since the implementation of the project was feasible and a followup for new requirements will be conducted. Given this, the learning outcome of the research was the best reward for the research team.

\section{Keywords}

C\# (computer programming language). Object-oriented programming (computing). Office automation. Data electronic processing.

El proceso de facturación que actualmente utiliza la librería $A B C$ es realizado de forma manual; el vendedor escribe con bolígrafo la cantidad de artículos adquiridos por el cliente y luego procede a calcular el total por pagar, auxiliándose de una calculadora o en ocasiones con base en cálculos propios. Este proceso tiene como objetivo lograr un medio sencillo para justificar las actividades económicas del negocio.

Sin embargo, el sistema de facturación actual de la librería $A B C$ tiene un uso ineficiente del tiempo. Emplear un prologando lapso en la realización de la factura manual contribuye a una lenta atención para los demás clientes. En caso de que haya un error en una factura, se tiene que anular; todo esto crea una imagen desfavorable del negocio. Además, el arqueo de caja se ve afectado, por su lenta realización, al tener que determinar manualmente el total vendido; sumatoria de todas las facturas realizadas.

La ineficiencia del sistema de facturación actual representa una pérdida financiera para la librería $A B C$. En promedio, la prontitud con la que un vendedor termina una factura, por cliente, varía de 3 a 5 minutos, determinada en parte por la cantidad de artículos vendidos o por la corrección de errores; ese tiempo puede reducirse significativamente, 
evitando así que los clientes tengan que esperar mucho su turno para ser atendidos y que terminen yéndose para adquirir los artículos de la competencia.

El proceso de facturación debe automatizarse para lograr más eficiencia en el tiempo de atención de los clientes y en la eliminación de errores en las operaciones de cobro al usar una calculadora.

El Código Tributario de El Salvador, según decreto 230, título III Deberes y Obligaciones Tributarias (Legislativa, 2000), Capítulo I, en la sección quinta, Art. 107, establece que "Ios contribuyentes del impuesto a la transferencia de bienes muebles y a la prestación de servicios están obligados a emitir y entregar, por cada operación, a otros contribuyentes un documento que, para los efectos de este código, se denominará 'Comprobante de Crédito Fiscal', que podrá ser emitido en forma manual, mecánica o computarizada", y que cuando se trate de operaciones realizadas con consumidores finales, deberán emitir y entregar, por cada operación, un documento que se denominará factura.

El beneficio social que involucra el proyecto de optimización de facturación, para la librería $A B C$, tiene como fin la generación de un sistema computacional que mejore el funcionamiento interno de un micronegocio, abriéndole una oportunidad de crecimiento al hacer más eficiente el proceso de facturación, a su vez, implica una oportunidad para los miembros del equipo de trabajo de adquirir más conocimiento en desarrollo de sistemas.

Requisitos previos de hardware y software para el sistema propuesto (IBM, 2010)

Tabla 1. Requisitos previos para el cliente, servidor de datos y sistema de facturación

\begin{tabular}{|c|c|}
\hline Requerimiento & Característica \\
\hline \multirow{2}{*}{ Sistema operativo } & • Windows Vista 7, 8 o 10 (cliente) \\
\hline & -Windows Vista, 7, 8 o 10, Windows Server 2008 o superior (servidor) \\
\hline \multirow{2}{*}{ Almacenamiento en disco } & - 1 GB disponible (Cliente) \\
\hline & - 5 GB disponible (servidor) \\
\hline \multirow{2}{*}{ RAM } & - 512 o superior (cliente) \\
\hline & - 1 GB o superior (servidor) \\
\hline Procesador & - Dual Core, Core 13 o superior \\
\hline \multirow[b]{2}{*}{ Requisitos previos (servidor) } & - Microsoft .NET Framework 2.0 \\
\hline & - El programa de instalación de SQL Server requiere el Service Pack 2 o superior \\
\hline \multirow{4}{*}{ Requisitos previos (Sistema de facturación) } & - NET Framework 2.0 para plataformas de 32 bits (x86) y plataformas de 64 bits (x64) \\
\hline & • Windows Installer 2.0 y 3.1 \\
\hline & - Bibliotecas en tiempo de ejecución de Visual C++ para procesadores IA64, x64 y x86 \\
\hline & - Visual Studio 2012 o superior \\
\hline Sistema gestor de base de datos & • SQL Server 2008 versión Express o superior \\
\hline Periféricos & - Teclado, mouse, impresor \\
\hline
\end{tabular}




\section{Lenguaje Visual C\# (Morales, 2011)}

En el proyecto se utilizó para el desarrollo del software de programación Microsoft C\#, el cual está diseñado para crear aplicaciones empresariales que se ejecutan en .NET Framework. Esto supone una evolución de Microsoft $\mathrm{C}$ y Microsoft C++; es sencillo, moderno, proporciona seguridad de tipos y está orientado a objetos. El código creado mediante C\# se compila como código administrado, significando que se beneficia de los servicios de Common Language Runtime. Estos servicios incluyen operar internamente entre lenguajes, recolección de elementos no utilizados, mejora de la seguridad y mayor compatibilidad entre versiones.

C\# se presenta como Visual C\# en el conjunto de programas Visual Studio .NET, y utiliza plantillas de proyecto, diseñadores, páginas de propiedades, asistentes de código, un modelo de objetos y otras características del entorno de desarrollo. La biblioteca para programar en Visual C\# es .NET Framework.

\section{Microsoft SQL Server 2012 (Mejia, 2010)}

Como gestor de bases de datos del sistema de facturación que se propuso, se utilizó Microsoft SQL Server 2012 Express, un sistema de administración de datos gratuito, eficaz y confiable que ofrece un almacén de datos completo y confiable para sitios web ligeros y aplicaciones de escritorio.

Microsoft SQL Server es un sistema de manejo de bases de datos del modelo relacional, desarrollado por la empresa Microsoft.

El lenguaje de desarrollo utilizado (por la línea de comandos o mediante la interfaz gráfica de Management Studio) es Transact-SQL (TSQL), una implementación del estándar ANSI del lenguaje SQL, utilizado para manipular y recuperar datos (DML), crear tablas y definir relaciones entre ellas (DDL).

\section{Ingeniería de software (Pressman, 2005)}

El término ingeniería de software fue introducido por primera vez a finales de 1960 en una conferencia destinada a su discusión, la cual fue posteriormente llamada crisis de software'. Esta crisis fue el resultado directo de la introducción del hardware de tercera generación computacional (Sommerville, 1989).
Para tener una idea clara de lo que es la ingeniería de software, vamos a definirlo en los dos puntos siguientes:

1. La aplicación de un enfoque sistemático, disciplinado y cuantificable hacia el desarrollo, operación y mantenimiento del software; es decir, la aplicación de ingeniería al software.

2. Es una disciplina o área de la Informática, o Ciencias de la computación, que ofrece métodos y técnicas para desarrollar y mantener software de calidad que resuelven problemas de todo tipo ([Pressman, 1998).

\section{Metodología orientada a objetos (Castellanos, 2009)}

Es así que, al estar hablando de objetos, es importante describir las ideas fundamentales implícitas en la tecnología orientada a objetos, entre las que están las siguientes:

- Objetos: cualquier cosa, real o abstracta, acerca de la cual almacenamos datos y aquellos métodos que los manipulan.

- Clase: es la implementación de un tipo de objeto. Especifica la estructura de datos y los métodos operacionales permitidos que se aplican a cada uno de sus objetos.

- Métodos: especifican la manera en la cual los datos de un objeto son manipulados.

- Los métodos, en un tipo de objeto, hacen solamente referencia a la estructura de datos de ese tipo de objeto. No deben de acceder directamente a la estructura de datos de otro objeto.

- Peticiones: solicitud de operaciones específicas; debe ser invocada usando uno o varios objetos como parámetros.

\section{Metodología de la investigación}

Método: científico experimental y observación científica

Participantes: el gerente general y los vendedores juntamente con el equipo investigador. 


\section{Instrumento y procedimiento}

\section{Técnica de la entrevista}

La entrevista se utiliza para obtener datos de forma oral, de manera que la persona entrevistada no tenga dificultad a la hora de aportar los datos correspondientes. En este caso, dicha entrevista se realizó de manera personal para tener la libertad de profundizar en lo investigado y para que el entrevistado aporte más detalles sobre el proceso de negocio, principalmente el de facturación.

Este proceso consistió en elaborar una serie de preguntas que la persona debía contestar con una respuesta abierta, garantizando que la información que daría sería confidencial; y su uso sería exclusivamente para fines académicos, en el caso de este proyecto. Esta entrevista se realizó por fases, las cuales se detallan a continuación:

Diseño de entrevista: se definieron los objetivos de la entrevista y se determinó a quienes se entrevistarían. En este caso se realizó la entrevista a dos vendedores y al dueño del negocio. El objetivo principal de la entrevista era conocer el proceso de facturación de dicho negocio.

Desarrollo de la entrevista: para el desarrollo de la entrevista, se acordó con el dueño del negocio el día y hora, para no obstaculizar el funcionamiento de la librería con los procesos y para tener disponibilidad suficiente para recabar detalles. Se realizaron anotaciones y grabaciones a los entrevistados para tener dos medios para el registro y posterior análisis de las respuestas.

Análisis e interpretación de datos: se recolectaron los datos necesarios, se codificaron y se llegaron a conclusiones, las cuales se tomarán como punto de partida para desarrollar una solución de software acorde con las necesidades del negocio.

\section{Técnica de la observación directa}

Se utilizó una segunda técnica de investigación: la observación directa. Esta permitió adquirir conocimiento acerca del problema en la librería $A B C$. El equipo de trabajo llegó al lugar y presenció de manera directa los procesos de facturación que realizan actualmente. A continuación, se explican los resultados que se obtuvieron a partir de esta técnica.
Mecanismos para mantener los flujos constantes de clientes

La librería visitada cuenta con un alto nivel de atención hacia los clientes; el servicio que los vendedores brindan es respetuoso, amable y muy profesional, puesto que, lejos antes de vender, se nota que primero asesoran al cliente con respecto a la diversidad de productos con los que cuentan y que son utilizados con el mismo fin. En algunos casos, la presentación de las vitrinas mantiene un aspecto limpio, los vendedores son muy ordenados en la distribución de la mercadería, de manera que resulta casi intuitivo saber dónde localizar un producto, a eso se le suma su horario de 7:00 am a 7:00 pm, permitiendo al público o clientes llegar al local a la hora que mejor les favorezca.

\section{Descripción y efecto del material de facturación}

El mecanismo y el proceso empleados para la generación del comprobante de compra es de forma manual, disponiendo de un talonario de factura, una calculadora y un lapicero. Para la realización de una factura se dedican de tres a cinco minutos; en ese corto tiempo se observó que algunos clientes optaban por retirarse del local al no ser atendidos con prontitud.

\section{Desventaja descubierta del material de facturación}

Durante el proceso, presenciamos la anulación de una a tres facturas. En algunos casos es debido a un mal cálculo aritmético efectuado por el vendedor; y en otros, por un error en la descripción de los artículos consignados en la factura. La consecuencia de estos eventos no es próspera, ya que el cliente no vinculaba una imagen favorable a la librería.

\section{Recomendaciones para mejorar la imagen y servicio}

En primera instancia, implementar un nuevo sistema de facturación automatizado para la librería $A B C$, que logre invertir dos minutos como máximo en el proceso de facturación por venta, y en segunda instancia, una mejoría en la atención y el despacho de los clientes, que se maximizarán de forma positiva, puesto que se logrará atender a un mayor número de clientes y evitará que se retiren y adquieran los artículos en otro negocio. 


\section{Resultados}

\section{Diseño de formularios}

Para mejorar el método de facturación en la librería $A B C$, se desarrolló un sistema automatizado de facturación que permitió eliminar el proceso manual que actualmente se realiza. Para el menú raíz de este sistema, se dispone de dos menús: Catálogos, que contiene los formularios que permiten la inserción de información inicial en la base de datos, los cuales tendrán disponibles los datos de clientes y productos, entre otros, para realizar posteriormente la factura; y Transacciones, que posee el formulario para realizar la facturación. En la figura 1, se observa el menú principal y el desglose del menú Catálogos.

Figura 1. Menú raíz del sistema de facturación
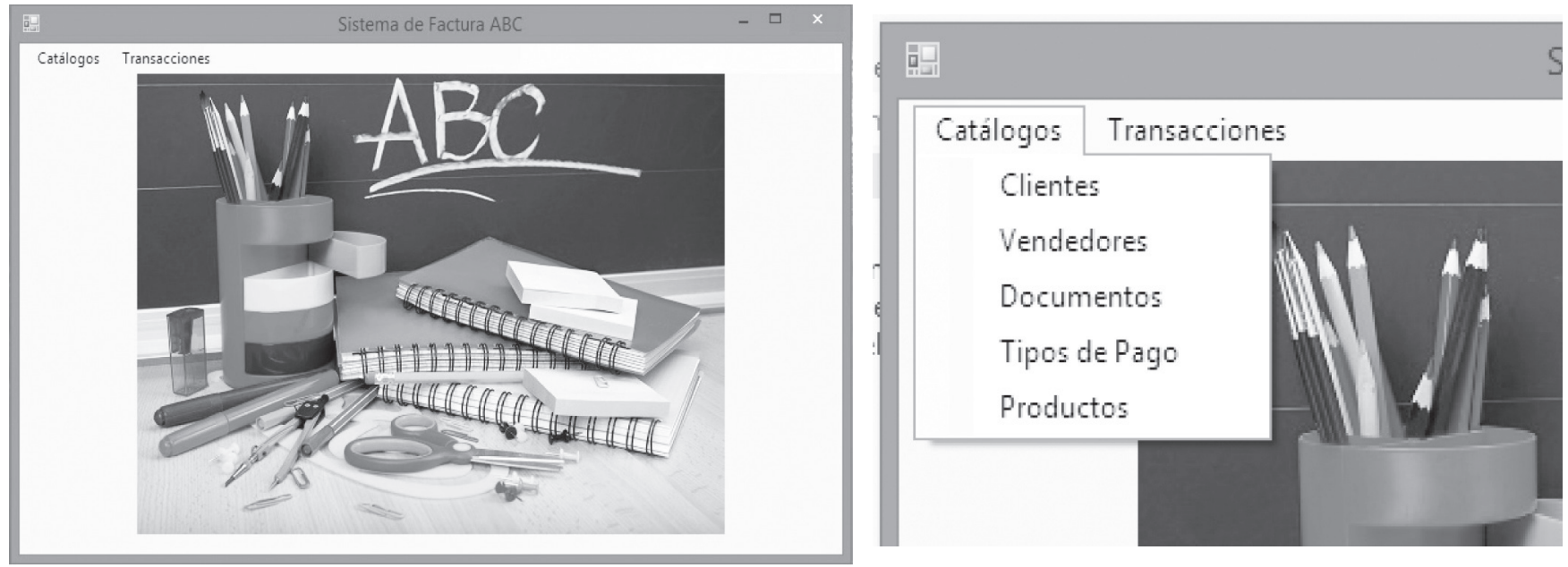

Fuente: elaboración propia.

\section{Tipos de pago}

El menú Catálogos lo componen los formularios siguientes:

- Clientes: el usuario podrá agregar, modificar, consultar o eliminar los datos de un cliente frecuente de la empresa. Se podrán ingresar datos como nombre, apellido, dirección, teléfono, e-mail, DUI.

- Vendedores: el usuario podrá agregar, modificar consultar o eliminar el registro de los vendedores que se encuentran trabajando en la librería. Los datos que se podrán capturar de estos son código, nombre del vendedor, número de Caja.

- Documentos: el usuario podrá agregar, modificar, consultar o eliminar los documentos que se dispondrán al momento de facturar, por ejemplo: crédito fiscal, factura de consumidor final o un tiquete. Si este último es el solicitado, entonces solo se añadirá el código, el nombre del documento y los correlativos inicial y final.

- Tipos de pago: el usuario podrá agregar, modificar, consultar o eliminar los tipos de pago, es decir, para especificar si el pago de la factura es al contado o al crédito. Únicamente se debe añadir el código y el nombre del documento, y, de ser necesario, especificar si el tipo de documento requiere cambio al momento de facturar.

- Productos: el usuario podrá agregar, modificar, consultar o eliminar los productos disponibles para la venta en la librería. De estos se ha de registrar el nombre, costo, precio, descripción, existencia mínima y máxima. 
Figura 2. Formulario de productos
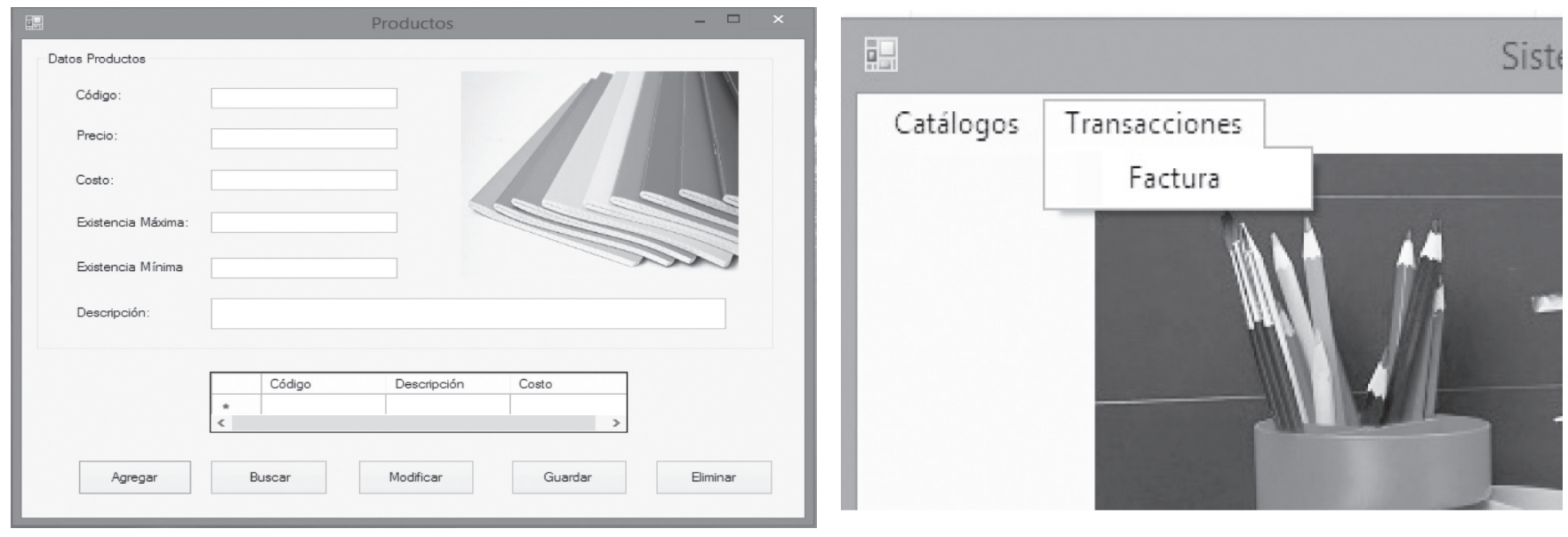

Fuente: elaboración propia.

Una vez definidos los catálogos del sistema, el usuario tiene las herramientas para realizar transacciones de facturación, en las que se podrá consultar la base de datos del cliente. Al contener los datos generales de un cliente frecuente, estos solo se añaden. En caso contrario, también se tendrá la opción de añadir manualmente a los nuevos clientes. Además, se podrán adicionar los productos que el cliente solicita consultándolos desde la base de datos; y el sistema automáticamente hará el cálculo para ser efectiva la venta.

El diseño del formulario de factura se presenta en la figura 3 , el cual se encuentra en el menú Transacciones dentro del sistema, que se presenta a continuación.

Figura 3. Menú Transacciones y Formulario de factura

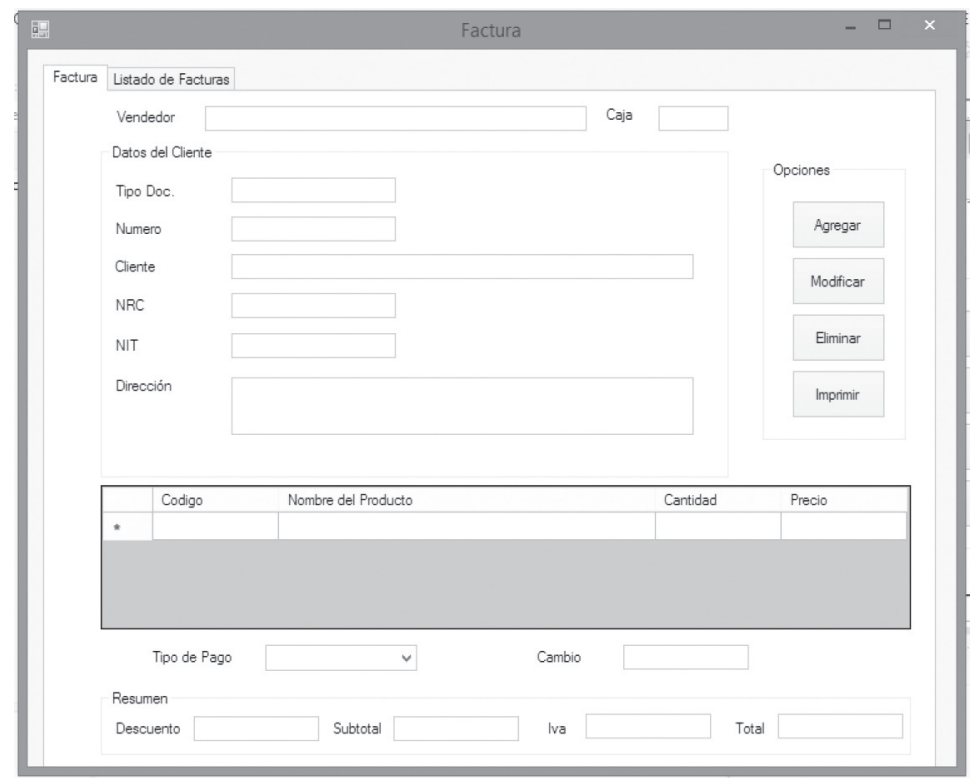

Fuente: elaboración propia. 


\section{Conclusión}

Debido a que el proceso de facturación que se utiliza en la librería $A B C$ es realizado de forma manual, se hace un ineficiente uso del tiempo y resulta en una lenta atención para los clientes, aparte de que es susceptible de cometer errores en la factura y que por eso sea necesario anularla. Además, el arqueo de caja se ve afectado en su efectividad por su lenta realización al tener que determinar de forma manual la sumatoria de todas las facturas realizadas para obtener el total de la venta del día.

Conociendo la problemática existente, mediante el análisis basado en la entrevista dirigida y en las observaciones directas sobre el proceso de negocio de la librería $A B C$, se determinó que el sistema de facturación actual provoca muchas desventajas, lo que genera un flujo de trabajo lento y en cierta forma obsoleto, por ende, se ve a simple vista la necesidad de crecer, mejorando y agilizando el proceso de venta mediante una facturación ágil. Como punto de partida para lograr este objetivo, es necesario la automatización de estos procesos de manera gradual, empezando con uno de los principales abastecimientos de información: la facturación. Esto les permitirá registrar sus facturas para cumplir con las disposiciones del Código Tributario, llevando un registro de facturación formal de acuerdo con la ley, y, por otro lado, agilizarán el proceso de atención al cliente y de realización de la venta para obtener una imagen más favorable para el negocio.

El sistema, en sí, será de mucha utilidad para la librería $\mathrm{ABC}$, porque le permitirá aprovechar el tiempo de atención al flujo de clientes, la reducción de márgenes de error en las los totales por pagar en las facturas y una forma más efectiva para garantizar a los auditores que los controles internos se llevan adecuadamente.
Esta automatización se realizó mediante un sistema en tiempo real, desarrollado basado en el lenguaje de programación C\#, el cual está diseñado para este tipo de aplicaciones, teniendo como gestor de base de datos SQL Server como un robusto repositorio de la información.

\section{Referencias}

Asamblea Legislativa. El Salvador. (2000). Asamblea Legislativa. Recuperado de http://www.asamblea. gob.sv/eparlamento/indice-legislativo/buscador-dedocumentos-legislativos/codigo-tributario

Castellanos, L. R. (2009). Desarrollo de sistemas de informacion: Bajo enfoque incremental. Recuperado de https://desarrollodesistemas.wordpress.com/ tag/ metodologia -orientada-a-objetos/

Corte Supre de Justica. El Salvador. (2000). Codigo tributario. Recuperado de http://www.oas.org/ juridico/ spanish/ mesicic3_slv_tributario.pdf

IBM. (2010). IBM Knowledge Center. Recuperado de https://www.ibm.com/support/ knowledgecenter/ es/SSAUB3_8.5.0/com.ibm.pt.help.doc/topics/r_ sysreqs_control_center.html

melvinbladimir5. (2010). Definiciones referentes a MySQL Server, SQL Server y Oracle. Recuperado de https://melvinbladimir5.wordpress.com/2010/02/11 /definiciones-referentes-a-mysql-server-sqlserver-y- oracle/

Morales, A. (2011). Visual Studio 2003 Retired Technical documentation. Recuperado de https://msdn. microsoft.com/es-sv/library/aa287558( v=vs.71). aspx

Pressman, R. (2005). Ingenieria de software. Recuperado de https://www.ecured.cu/Ingenier\%C3 \%ADa_ de_software 